\section{Fallbuch WISC-V - Die Wechsler Intelligence Scale for Children - Fifth Edition in der Praxis}

Monika Daseking, Franz Petermann (Hrsg.). Fallbuch WISC-V. 5. Praxisausgabe des Wechsler Intelligenztests (Wechsler Intelligence Scale for Children). Göttingen: Hogrefe Verlag, 2021; 351 Seiten, ISBN 9783801730086, 34,95 Euro

Die Intelligenztestung und Testung auf kognitive Fähigkeiten bei Kindern und Jugendlichen spielt seit Jahrzehnten eine große Rolle, um ein möglichst gutes Beschulen, eine gute Bildung und eine gute Förderung von Kindern und Jugendlichen zu gewährleisten. Ohne Testverfahren überbewerten zu wollen, muss konstatiert werden, dass der früher als Hamburg-Wechsler-Intelligenztest für Kinder bezeichnete Wechsler-Intelligenztest eine wichtige Funktion für die Weichenstellung für die Beschulung von Kindern und den weiteren Bildungsgang der Kinder darstellt. Inzwischen sind auch digitale Alternativen zur herkömmlichen Papierform der Testformate vorhanden. Das jetzt hier vorliegende Buch versetzt aber Pädagogen, Psychologen und Kinderärzte in die Lage, die Möglichkeiten, aber auch die Limitierungen der Intelligenztestung zu erlernen. Themen wie Intelligenztestungen beim Vorhandensein eines Migrationshintergrundes, besondere Themen wie Epilepsie, Hirntumoren und Syndromen werden besprochen und die Anwendung des Wechsler Intelligenztestes gerade zur Diagnostik, z. B. von intellektu- eller Hochbegabung, von Intelligenzminderung sowie Lese- und Rechtschreibstörungen und Rechenthemen, einzuordnen. Das Buch ist entsprechend unverzichtbar. Eine Lektüre für Berufe, die sowohl Intelligenztests als Grundlage ihres Handelns identifizieren müssen als auch für die Testenden selbst.

Sehr bedauerlich und traurig ist, dass der Herausgeber Franz Petermann leider zu früh verstorben ist und vermutlich die Druckausgabe seines neuesten Buches nicht mehr miterleben durfte. Wir sind ebenso wie Professorin Monika Daseking traurig über diese Tatsache.

Wieland Kiess, Leipzig 